\title{
Experimental investigation of turbulent flow past four grooved and smooth cylinders in an in-line square arrangement
}

\author{
O. Ladjedel ${ }^{1}$, M.Bouacha ${ }^{1}$, L. Adjlout ${ }^{1 \mathrm{a}},{ }^{1}$ T.Yahiaoui $^{2}$ and N. Belharat ${ }^{1}$ \\ ${ }^{1}$ Laboratoire d'aérohydrodynamique naval, Département de Génie Maritime, Mechanical Engineering Faculty, \\ USTO Oran, Oran 31000 Algeria \\ ${ }^{2}$ Laboratoire d'aéronautique et systèmes propulsive Département de Génie Mécanique Mechanical Engineering \\ Faculty, USTO Oran, Oran 31000 Algeria
}

\begin{abstract}
An experimental study of turbulent flow past four cylinders in square arrangement with a space ratio of $(T / D=P / D=2.88)$ is performed. The investigation focuses on effects of Reynolds number and the shape of cylinders on the force and pressure coefficients of the cylinders. Two cases are investigated: four smooth cylinders (case1) and four grooved cylinders (case2). The cylinders are equipped with two grooves placed on the external surface at $90^{\circ}$ and $270^{\circ}$ degrees. The pressure distributions along the tubes $(22$ circumferential pressure taping) were determined for a variation of the azimuthal angle from 0 to $360 \mathrm{deg}$. The drag and lift forces are measured using the TE 44 balance. The results show a bistable flow often exists behind the downstream cylinders is observed. By rising the Reynolds number the pressure coefficient increases in the absolute value.
\end{abstract}

\section{Introduction}

The wake interference around the cylinder arrays has been widely investigated in the past because of its inherent importance and practical significance in engineering applications such as heat exchangers, offshore structures, and even micro-electro-mechanical systems.

Over the past years, several studies on flow around four-cylinder arrays have been investigated using experimental and numerical techniques. To understand effects of the spacing ratio on flow patterns and vortex shedding frequency, Lam and Lo [1] and Lam et al. [2] performed visualization studies. Sayers [3, 4] conducted experiments on four cylinders in a square configuration in the spacing ratio range of 1.55 with an aspect ratio of 11.5 at Reynolds number of 30000 . The pressure distributions and the lift/drag coefficients on four cylinders with the spacing ratio varying from 1.26 to 5.8 are measured by Lam and Fang [5] and Lam et al. [6]. Lam and Zou [7] further investigated effects of the spacing ratio and Reynolds number on mean velocity distribution and flow patterns around the four in-line cylinders at subcritical Reynolds numbers using laser Doppler anemometer (LDA) and digital particle image velocimetry (DPIV) techniques.

Due to the difficulties in data acquisition in certain positions and configurations in the experimental study, numerical simulation results for such complex turbulent flows have been sought. Numerical simulations of the flow around one or two cylinders have been reported in the past for turbulent flows. However, there are still relatively little numerical investigations on the fourcylinder arrays. Farrant et al. [8] captured twodimensional (2-D) flow characteristics and interactive forces associated with flow around four equispaced cylinders for two orientations (in-line arrangement and an alignment angle of $45^{\circ}$ arrangement ) for spacing ratios $\mathrm{L} / \mathrm{D}=3$ and 5 at $\mathrm{Re}=200$ using a cell boundary element method. With the typical case of four cylinders being arranged with alignment angle of $45^{\circ}$ and the case of inline arrangement at $\mathrm{L} / \mathrm{D}=5$, the computation results showed agreement with visualization results obtained by Lam and Lo [1] and Lam et al [2, 6]. However, it is interesting to note that for the in-line arrangement with $\mathrm{L} / \mathrm{D}=3$, the computational results showed that shear layers from upstream cylinders roll up into mature vortices and impinge on the downstream cylinders' surface. Lam et al. [2] reconfirmed that accurate prediction of the shielding flow pattern for the critical arrangement is a challenge for numerical simulation. Moreover, Lam et al. [9] failed to simulate the shielding flow pattern for the same arrangement. This indicates that there is a large discrepancy between experimental measurements and computational results at the critical spacing ratios. It should be noted that all the numerical simulations above were carried out by assuming 2-D flow around the four cylinders, in spite of the actual 3-D flow around them. For an adequate representation of flow around four cylinders, a relatively time-consuming 3-D simulation should be carried out. Lam and Zou [7] employed 3-D large eddy simulation on turbulent cross-

\footnotetext{
adjloutl@yahoo.fr
} 
flow around four in-line cylinders with different pacing ratios at a subcritical Reynolds number. The numerical results predict accurately some of the wake flow characteristics around four cylinders and agree with the experimental measurements. The purpose of the present work is to investigate experimentally the influence and the interest of using grooves on flow around four cylinders in square arrangement.

\section{Experimental set up}

The experiments are carried out in closed circuit wind tunnel. The test section is of square cross-section $0.46 \mathrm{~m} \times$ $0.46 \mathrm{~m}$ and $1.2 \mathrm{~m}$ long. The closed circuit wind tunnel is of conventional design in which the velocity could reach 60 $\mathrm{m} / \mathrm{s}$ and the turbulence intensity is less than $1 \%$.

The test section is composed of four rigid PVC tubes in square arrangement with an identical external diameter of $40 \mathrm{~mm}$ and $460 \mathrm{~mm}$ length as shown in figure1.

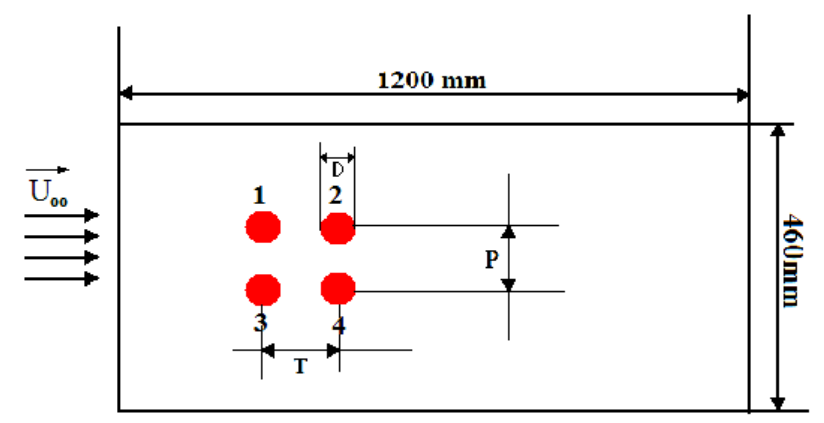

Figure 1. Experimental setup.

The grooved cylinder is equipped with 22 pressure tapings on its circumference and one pressure taping for the smooth cylinder where the latter will be turned from $0^{\circ}$ to $360^{\circ}$ with an interval of $10^{\circ}$. The diameter of the tapings is $0.5 \mathrm{~mm}$ and the location of the tapings for both cylinders is at mid-span. The shape and dimensions of the groove are shown in Figure (2). The pressure measurements are carried out for the upstream and the downstream cylinder for an azimuthal angle from $0^{\circ}$ to $360^{\circ}$. In the present work, the drag forces are measured using the TE81 balance and the pressure distributions on the cylinder were performed using a TE44DPS differential pressure scanner. The latter pressure scanning box allowed sequential selection of up to 20 pressure tapings. The display unit links to a computer, loaded with DATASLIM software for data analysis and logging of results. For details of the wind tunnel and the experimental setup (see Ladjedel et al. [10]).

\section{Results and discussion}

Two cases are investigated; for the first case all cylinders are smooth and for the second one grooved cylinders are used. The experiment includes two main parts: the first one is measuring the pressure around the cylinder for three Reynolds number: $\mathrm{Re}_{1}=2 \times 10^{4,} \mathrm{Re}_{2}=5 \times 10^{4}$ and $\mathrm{Re}_{3}=7 \times 10^{4}$ (based on diameter and Free stream velocity); the second one is measuring drag forces for various Reynolds number. The spacing between cylinders: $T / D=P / D=2.88,(\mathrm{D}=40 \mathrm{~mm}, T / D$ and $P / D$ are the transverse and longitudinal pitch-to-diameter ratio).

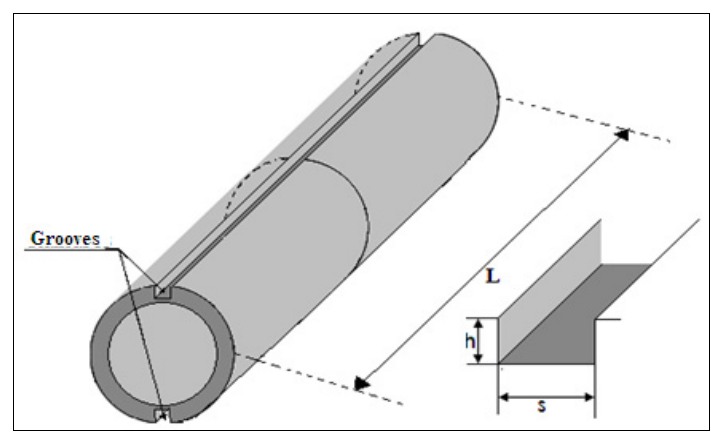

Figure 2. Shape and dimensions of the grooves.

Measurement starts from the frontal point facing the mainstream flow and ends at the same point after a complete revolution. The average value is then calculated. The pressure coefficient is defined as:

$$
C_{P}=\frac{p-p_{r e f}}{0.5 \times\left(\rho \times U_{\infty}^{2}\right)}
$$
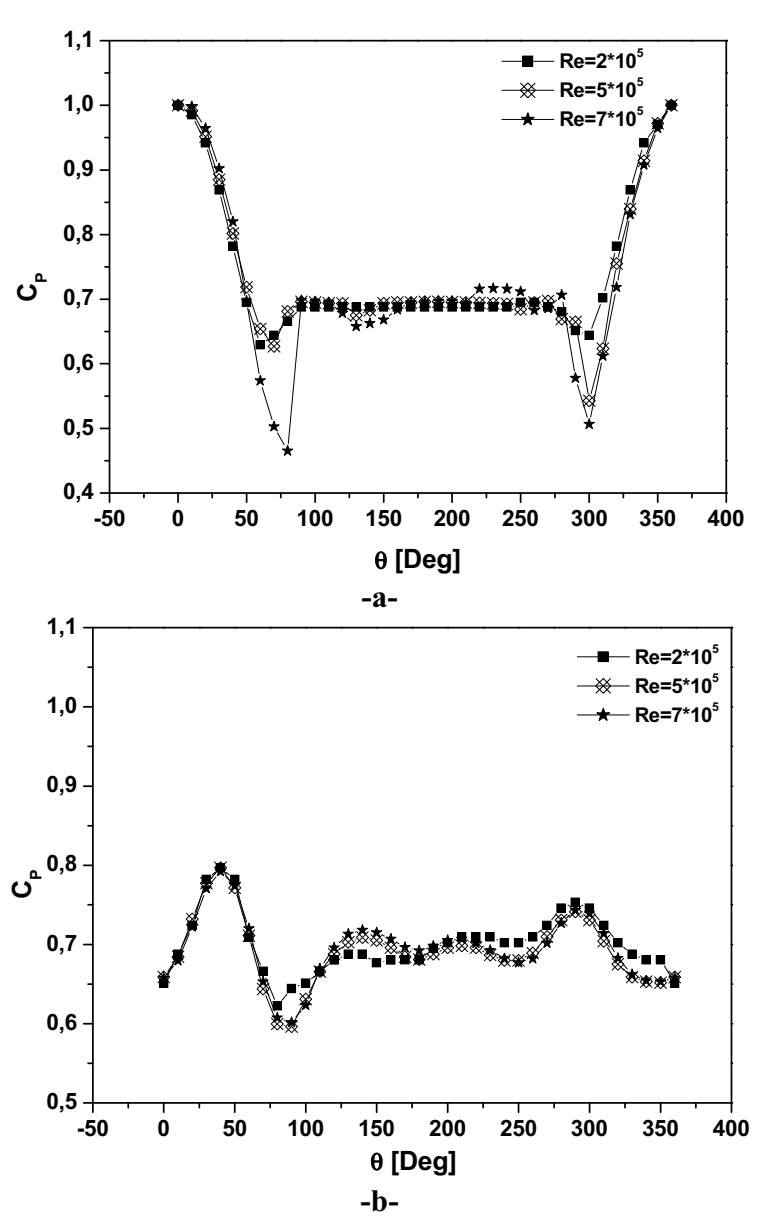

Figure 3. Mean pressure coefficient distribution for the first case. (a) Cylinder 1, (b) Cylinder 2.

Figure 3 and 4 show the mean pressure distribution at the mid-spam for the two studied cases. Three Reynolds 
number are tested. On the whole, the pressure distributions on cylinder 1 (Figure $3 \mathrm{a}$ and $4 \mathrm{a}$ ) seem to be similar to that of an isolated cylinder. In figure 3(a) a large peak was observed at $280^{\circ}$ angle for Reynolds numbers $\operatorname{Re}=7 \times 10^{5}$ and $5 \times 10^{5}$. The stagnation point on cylinder 1 is around $\theta=0^{\circ}$ for the first case and shifts from the frontal position $\theta=0^{\circ}$ to the inner side $(\theta=$ $30^{\circ}$ ). Figure $4 \mathrm{~b}$ and $5 \mathrm{~b}$ illustrate that for the aspect ratio studied the interaction between the cylinders appears to be non-negligible. A bistable flow exists behind cylinder 2 with an alternate wide wake and a narrow wake. The lowest pressure coefficient in the pressure recovery region corresponds to a wide bistable wake behind cylinder 2 . In general it is observed that the pressure coefficient increases in the absolute value by the rising of Reynolds number.
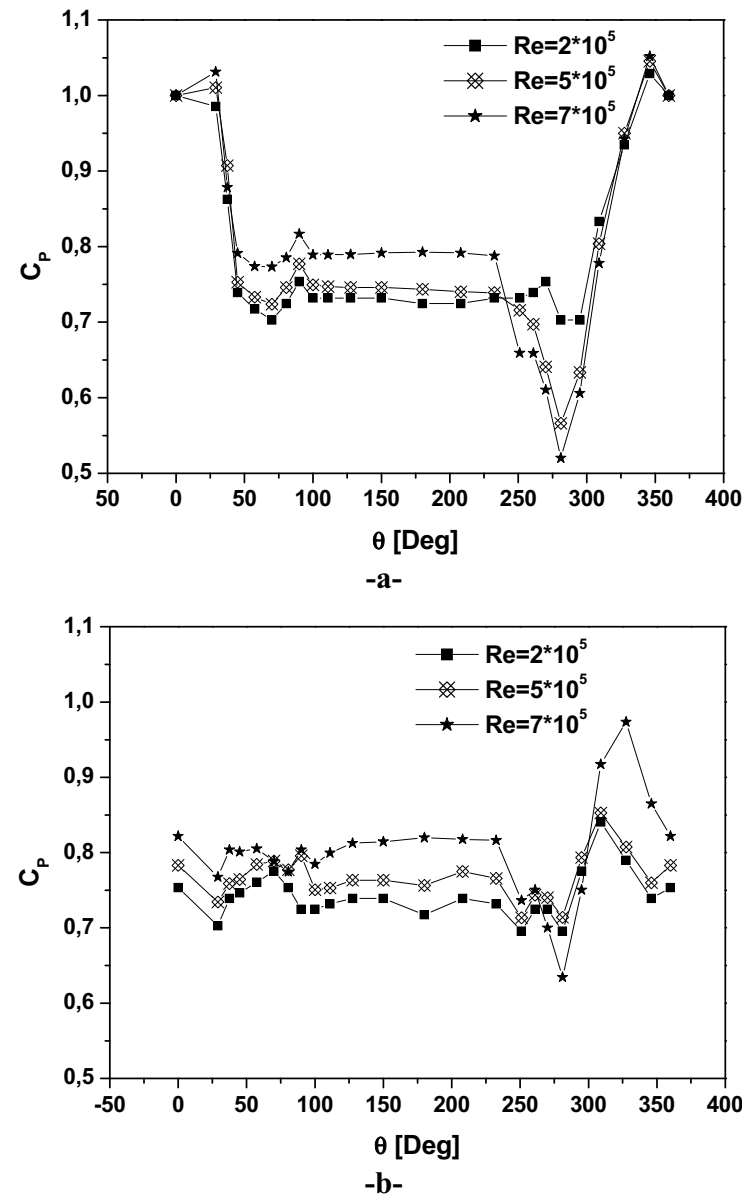

Figure 4. Mean pressure coefficient distribution for the second case. (a) Cylinder 1, (b) Cylinder 2.

The drag coefficient is calculated using the following formula:

$$
C_{D}=\frac{2 D}{\rho \times U_{0}^{2} \times S}
$$

D: Drag force $(\mathrm{N})$,

S: Projected area of the cylinder $S=d \times L$

$\rho$ : Fluid density $\left(\mathrm{Kg} \mathrm{m}^{-3}\right)$.

$\mathrm{L}$ : cylinder Length (m).

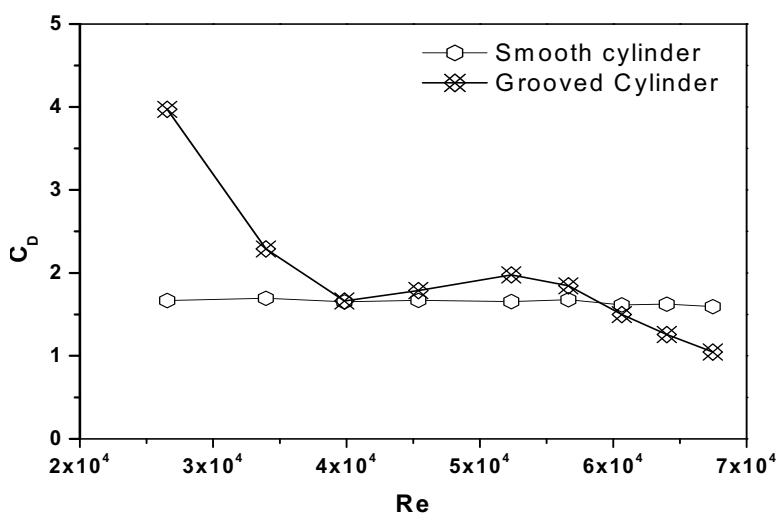

Figure 5. Drag coefficients distributions for the position 1.

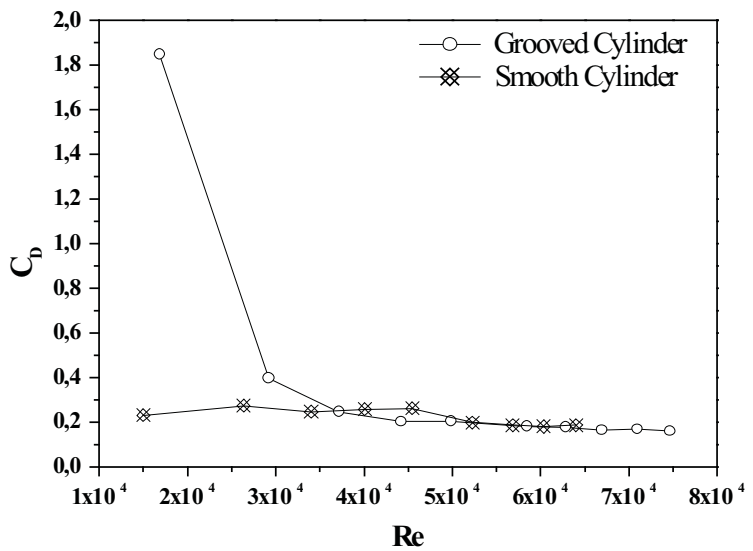

Figure 6. Drag coefficients distributions for the position 2.

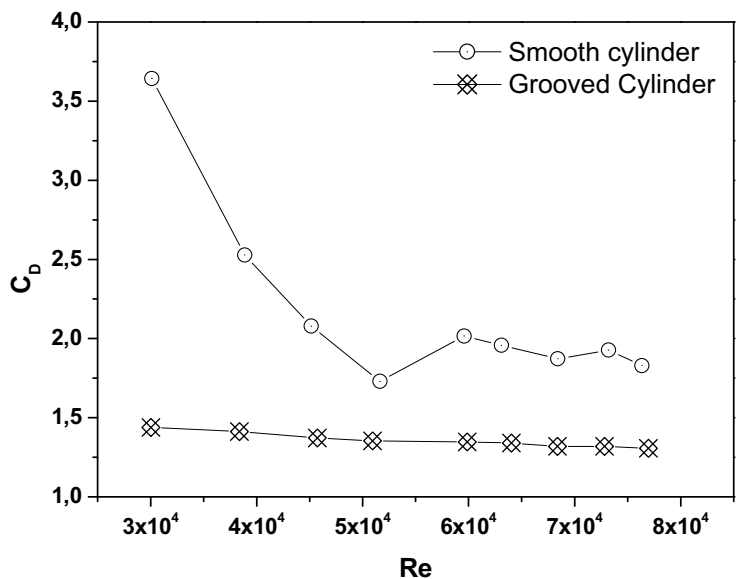

Figure 7. Drag coefficients distributions for the position 3.

Figures 5, 6, 7 and 8 show respectively a comparison of drag evolution between the smooth and the grooved cylinder situated at the position 1,2,3 and 4. It is observed that for the grooved cylinder the values of drag coefficient are almost constant compared with smooth one. It is observed that the upstream cylinders (tube 1 and 3) have a greater drag pressure compared to the downstream cylinders (tube 2 and 1) because the first cylinder is directly opposed to the flow inlet. However, the second one is in the wake of the latter. For low Reynolds number the drag force is greater in the first studied case (smooth cylinder) and this compared to the 
second case (grooved cylinder). This remark is in agreement with Ladjedel et al. [10] in their experimental investigation. They found that by using grooved cylinder the drag force is reduced for higher Reynolds (greater than $3.2 \times 10^{4}$ ).

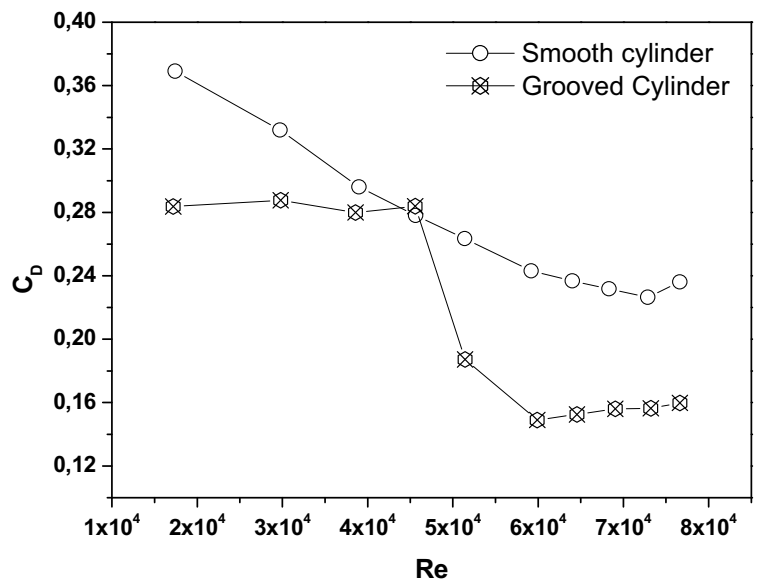

Figure 8. Drag coefficients distributions for the position 4.

\section{Conclusions}

The flow around four cylinders in an in-line square configuration is investigated experimentally. The Effect of grooved cylinder is investigated. The mean pressure and drag force where obtained for the three Reynolds number. From the pressure distribution a bistable flow often exists behind the downstream cylinder is observed. The pressure coefficient increases in the absolute value by the rising of Reynolds number. As remarked in the previous work the upstream cylinders has a greater drag pressure compared to the downstream cylinder. Finally, by using the grooved cylinder the drag force is reduced for this configuration of four equispaced cylinders in an in-line array.

\section{References}

1. Lam, K., Lo, S.C., Journal of Fluids and Structures 6, 109-131 (1992)

2. Lam, K., Li, J.Y., Chan, K.T., So, R.M.C., Journal of Fluids and Structures 17, 579-665 (2003a)

3. Sayers, A.T. Journal of Wind Engineering and Industrial Aerodynamics 31, 9-28 (1988)

4. Sayers, A.T., Journal of Wind Engineering and Industrial Aerodynamics 34, 213-221(1990).

5. Lam, K., Fang, X. Journal of Fluids and Structures 9, 195-214 (1995)

6. Lam, K., Li, J.Y., So, R.M.C., Journal of Fluids and Structures 18, 305-324 (2003b)

7. Lam, K., Zou, L., Journal of Mechanical Science and Technology 21, 1338-1343 (2007)

8. Farrant, T., Tan, M., Price, W.G., Journal of Fluids and Structures 14, 375-402 (2000)

9. Lam, K., Gong, W.Q., So, R.M.C., Journal of Fluids and Structures 24, 34-57 (2008)
10. Omar Ladjedel, Lahouari Adjlout, Tayeb Yahiaoui, and Omar Imine. Mechanics \& Industry 14, 287297DOI: 10.1051/meca/2013066 (2013) 